\title{
Immunological identification of Neisseria gonorrhoeae with monoclonal and polyclonal antibody coagglutination reagents
}

\author{
H YOUNG, KATHERINE G REID
}

From the STD Diagnostic Laboratory, Department of Bacteriology, University of Edinburgh Medical School, Edinburgh EH8 $9 A G$

SUMMARY The reliability of immunological identification of Neisseria gonorrhoeae using polyclonal and monoclonal antibody coagglutination reagents has been evaluated. When clinical isolates of neisseriae were tested in an "in use" trial the sensitivity and specificity of each reagent were similar and the overall agreement with carbohydrate utilisation was $97.9 \%(141 / 144)$ for the polyclonal antibody reagent and $97 \cdot 2 \%(140 / 144)$ for the monoclonal reagent. When results of testing 13 stock cultures of $N$ lactamica and five stock cultures of $\beta$-lactamase producing Branhamella catarrhalis were combined with the results for clinical isolates of non-gonococcal neisseriae the agreement with carbohydrate utilisation was $86.5 \%$ (64/74) for the polyclonal reagent and $97 \cdot 3 \%(72 / 74)$ for the monoclonal reagent: this difference is statistically significant at the $5 \%$ level.

Calculation of positive and negative predictive values showed differences in the reliability of the coagglutination reagents when testing Gram negative diplococci isolated from various anatomical sites. The value and limitations of the polyclonal and monoclonal reagents were similar with respect to anogenital isolates: $N$ gonorrhoeae was confirmed by a positive result but not excluded by a negative result. The monoclonal reagent was superior for testing throat isolates; although a negative result with either reagent confirmed Gram negative diplococci as non-gonococcal neisseriae, a positive result with the monoclonal reagent was more reliable (predictive value $93 \%$ ) than a positive result with the polyclonal reagent (predictive value $86 \%$ ).

Gonorrhoea remains a prevalent infection; 55784 cases were identified and treated in clinics in England during the year ending 30 June $1980 .^{1}$ Because of the heavy microbiological workload this imposes there is a considerable incentive to develop and implement simple, rapid, and reliable methods of differentiating between gonococcal and nongonococcal neisseriae. The development of the coagglutination reaction ${ }^{2}$ increased the popularity of immunological identification of Neisseria gonorrhoeae and coagglutination has now superseded immunofluorescence in many laboratories. ${ }^{3-5}$ Some immunological cross reactions are found between common antigens on $N$ gonorrhoeae and nongonococcal neisseria. Such reactions are not unexpected since the polyclonal antibodies used in the coagglutination reagent, although absorbed to

Accepted for publication 8 August 1984 remove non-reacting antibodies, are prepared by immunising rabbits with whole cells of several strains of gonococci. The cross reactions limit the usefulness of coagglutination in identifying isolates from sites such as the throat, where $\boldsymbol{N}$ meningitidis and to a lesser extent $N$ lactamica are the most common isolates, ${ }^{6}$ and the anorectum of homosexual men, where meningococci are not uncommon. ${ }^{7}$

Monoclonal antibodies which recognise a single antigenic determinant should facilitate the production of more specific diagnostic reagents. We have evaluated the immunological identification of $N$ gonorrhoeae with coagglutination reagents which employ monoclonal antibodies produced against the major outer membrane protein (protein I) of $N$ gonorrhoeae. ${ }^{8}$

\section{Material and methods}

Clinical isolates of neisseriae were obtained from 1276 
patients attending the department of genitourinary medicine of the Royal Infirmary. Anogenital and pharyngeal material was inoculated directly on to Modified New York City (MNYC) medium ${ }^{9}$ and transported to the laboratory within $4 \mathrm{~h}$. After overnight incubation at $36^{\circ} \mathrm{C}$ in air enriched with carbon dioxide $(5-10 \%)$ cultures were screened for oxidase positive Gram negative diplococci (GNDC). Suspected neisserial colonies (oxidase positive GNDC) were subcultured on to MNYC medium and incubated overnight before carrying out the rapid carbohydrate utilisation' and coagglutination tests. If more than one anogenital site from a female patient yielded Gram negative diplococci, cultures from one site only, usually the cervix, were tested by coagglutination.

In the first part of the study all Gram negative diplococci were tested in parallel with polyclonal and monoclonal coagglutination reagents. Thirteen stock cultures of $\boldsymbol{N}$ lactamica and five of $\beta$-lactamase producing Branhamella catarrhalis were also tested with both types of reagent. During the second half of the study only monoclonal reagents were used, and test performance was compared with bacterial suspensions prepared by boiling and by treatment with antigen releasing agent.

\section{COAGGLUTINATION}

Coagglutination with polyclonal antibody reagents (Phadebact Gonococus test, Pharmacia Diagnostics AB, Sweden) was performed with a boiled suspension of organism as described elsewhere ${ }^{5}$ except that a $20 \mu l$ unit volume was used.

Coagglutination with monoclonal reagents (GONO GEN, New Horizons Diagnostic Company, Columbia, MD) was carried out with boiled suspensions of organisms and with suspensions treated with an antigen releasing agent available from the same source.

\section{BOILED SUSPENSION METHOD}

A heavy suspension of each culture to be tested was made by removing the growth from the culture plate with a cotton tipped swab and emulsifying it in $\mathbf{0 . 2}$ $\mathrm{ml}$ of distilled water in a tube. The tube was covered and the suspension heated in a boiling water bath for $5 \mathrm{~min}$. After cooling to room temperature $20 \mu \mathrm{l}$ aliquots of suspension were mixed with an equal volume of test reagent (murine monoclonal antiprotein I antibody bound to dead staphylococci) and control reagent (non-immune rabbit IgG bound to dead staphylococci) on a clear glass slide. Three tests were carried out on a single slide $(7.6 \mathrm{~cm} \times 5.0$ $\mathrm{cm})$ with six clearly defined areas. The slide was rocked gently in a rotary fashion for $1 \mathrm{~min}$ and the results read against a dark background using indirect light.

ANTIGEN RELEASING AGENT

Suspensions of each test organism were made on a glass slide by mixing several colonies with $20 \mu$ l volumes of antigen releasing agent (diluted 1/5 with distilled water). Test reagent $(20 \mu \mathrm{l})$ and control reagent $(20 \mu \mathrm{l})$ were mixed with the suspensions and the slides rocked and read as above.

Results were interpreted as follows:

Positive - clumping or agglutination with test reagent significantly stronger than with control reagent. Equivocal-clumping or agglutination slightly stronger with test reagent than with control reagent.

Non-specific-reaction of equal strength with test and control reagents.

Negative - no reaction with the test reagent irrespective of any reaction with the control reagent.

Calculation of sensitivity and specificity were as follows:

Sensitivity-percentage of gonococcal isolates by carbohydrate utilisation that were coagglutination positive.

Specificity-the percentage of non-gonococcal isolates by carbohydrate utilisation that were coagglutination negative.

Predictive values were calculated according to the formula given by Veehio. ${ }^{10}$

The predictive value of a positive test $\left(\mathrm{PV}^{+}\right)$is the probability that the Gram negative diplococci giving a positive coagglutination test are gonococci and is calculated according to the formula:

$$
\mathrm{PV}^{+} \frac{\mathrm{pa}}{\mathrm{pa}+(1-\mathrm{p})(1-\mathrm{b})} \times 100
$$

The predictive value of a negative test $\left(\mathrm{PV}^{-}\right)$is the probability that the Gram negative diplococci giving a negative coagglutination test are nongonococcal neisseria (or Branhamella) and the formula is:

$$
\mathrm{PV}^{-} \frac{(1-p) b}{(1-p) b+p(1-a)} \times 100
$$

where $p=$ prevalence of gonococcal Gram negative diplococci within the total population of Gram negative diplococci isolated from a given site, $a=$ test sensitivity, and $b=$ test specificity.

The significance of differences in the results was determined by the $\chi^{2}$ test with Yates' correction.

\section{Results}

Table 1 shows the results of polyclonal and mono- 
Table 1 Results of polyclonal and monoclonal antibody coagglutination reagents tested with 144 clinical isolates of neisseriae.

\begin{tabular}{|c|c|c|c|c|c|c|c|}
\hline \multirow{2}{*}{$\begin{array}{l}\text { Identity by } \\
\text { carbohydrate } \\
\text { utilisation }\end{array}$} & \multirow[t]{2}{*}{ No of isolates } & \multicolumn{3}{|c|}{ Polyclonal } & \multicolumn{3}{|c|}{ Monoclonal } \\
\hline & & Positive & Non-specific & Negative & Positive & Non-specific & Negative \\
\hline $\begin{array}{l}N \text { gonorrhoeae } \\
N \text { meningitidis } \\
N \text { lactamica } \\
N \text { perflava } \\
\text { Total }\end{array}$ & $\begin{array}{r}88 \\
52 \\
3 \\
1 \\
144\end{array}$ & $\begin{array}{r}88 \\
0 \\
2 \\
0 \\
90\end{array}$ & $\begin{array}{l}0 \\
0 \\
1 \\
0 \\
1\end{array}$ & $\begin{array}{r}0 \\
52 \\
0 \\
1 \\
53\end{array}$ & $\begin{array}{c}86 \\
1^{*} \\
0 \\
0 \\
87\end{array}$ & $\begin{array}{l}1 \\
0 \\
1 \\
0 \\
2\end{array}$ & $\begin{array}{r}1 \\
51 \\
2 \\
1 \\
55\end{array}$ \\
\hline
\end{tabular}

*Negative after two subcultures.

Table 2 Monoclonal antibody coagglutination test applied to suspensions of 171 neisserial isolates prepared by boiling and by treatment with antigen releasing agent

\begin{tabular}{|c|c|c|c|c|c|}
\hline \multirow{2}{*}{$\begin{array}{l}\text { Identity by } \\
\text { carbohydrate } \\
\text { utilisation }\end{array}$} & \multirow[t]{2}{*}{ No of isolates } & \multicolumn{4}{|c|}{ Results with suspensions prepared by boiling (and by treatment with releasing agent) } \\
\hline & & Positive & Equivocal & Non-specific & Negative \\
\hline $\begin{array}{l}N \text { gonorrhoeae } \\
N \text { meningitidis } \\
N \text { lactamica } \\
N \text { perflava } \\
\text { Total }\end{array}$ & $\begin{array}{r}117 \\
51 \\
2 \\
1 \\
171\end{array}$ & $\begin{array}{c}112(95) \\
0(1) \\
0(0) \\
0(0) \\
112(96)\end{array}$ & $\begin{array}{l}1(10) \\
0(8) \\
0(2) \\
0(0) \\
1(20)\end{array}$ & $\begin{array}{l}2(1) \\
0(0) \\
0(0) \\
0(0) \\
2(1)\end{array}$ & $\begin{array}{r}2(11) \\
51(42) \\
2(0) \\
1(1) \\
56(54)\end{array}$ \\
\hline
\end{tabular}

Table 3 Predictive values of monoclonal and polyclonal antibody coagglutination reagents for Gram negative diplococci isolated from various sites

\begin{tabular}{|c|c|c|c|c|c|}
\hline \multirow{2}{*}{$\begin{array}{l}\text { Source of Gram } \\
\text { negative diplococci }\end{array}$} & \multirow{2}{*}{$\begin{array}{l}\text { Proportion of } \\
\text { gonoccocal Gram } \\
\text { negative diplococci }\end{array}$} & \multicolumn{2}{|c|}{ Positive predictive value } & \multicolumn{2}{|c|}{ Negative predictive value } \\
\hline & & Monoclonal & Polyclonal & Monoclonal & Polyclonal \\
\hline \multicolumn{6}{|c|}{$\begin{array}{l}\text { Female anogenital and } \\
\text { heterosexual male }\end{array}$} \\
\hline $\begin{array}{l}\text { Homosexual male } \\
\text { anogenital }\end{array}$ & 0.953 & 99.91 & 99.80 & $58 \cdot 75$ & $64 \cdot 58$ \\
\hline $\begin{array}{l}\text { Homosexual male } \\
\text { rectum }\end{array}$ & 0.920 & $99 \cdot 84$ & $99 \cdot 65$ & $71 \cdot 48$ & $76 \cdot 27$ \\
\hline $\begin{array}{l}\text { Throat (all patient } \\
\text { groups) }\end{array}$ & $0 \cdot 203$ & $93 \cdot 18$ & $86 \cdot 42$ & $99 \cdot 13$ & $99 \cdot 32$ \\
\hline
\end{tabular}

clonal antibody coagglutination results with boiled suspensions of clinical isolates of neisseriae. The sensitivity and specificity of the monoclonal reagent were $97.7 \%(86 / 88)$ and $96.4 \%(54 / 56)$, respectively, compared with values of $100 \%(88 / 88)$ and $94.6 \%(53 / 56)$ for the polyclonal reagent. The overall agreement with carbohydrate utilisation was $97.9 \%(141 / 144)$ for the polyclonal antibody reagent and $97.2 \%(140 / 144)$ for the monoclonal reagent. These differences are not significant at the $5 \%$ level.

Whereas all 13 stock cultures of $N$ lactamica were negative with the monoclonal reagent seven gave a positive result with the polyclonal reagent. All five isolates of $\beta$-lactamase producing $B$ catarrhalis were negative with both reagents. When these results were combined with those for clinical isolates of non-gonococcal neisseriae the agreement with carbohydrate utilisation was $97.3 \%(72 / 74)$ for the monoclonal reagent and $86.5 \%(64 / 74)$ for the polyclonal reagent. This is a significant difference $\left(\chi^{2}=4.5 ; p<0.05\right)$.

Results of the monoclonal reagent with bacterial suspensions prepared by boiling and by treatment with antigen releasing agent are given in Table 2 . Suspensions prepared with releasing agent tended to be stringy compared with the uniform suspensions obtained by boiling. On many occasions the stringy nature of the suspensions made it impossible to score results unequivocally positive or negative. The overall correlation with carbohydrate utilisation was $97.1 \%(166 / 171)$ for the test with boiled suspension and $80.7 \%(138 / 171)$ when antigen releasing agent was used. This is a highly significant difference $\left(\chi^{2}=\right.$ $21.6 ; p<0.001)$. The difference in test performance remains significant when gonococcal $(p<0.01)$ and non-gonococcal neisseriae $(p<0.001)$ are considered separately. 
Calculation of predictive values

The positive and negative predictive values of the polyclonal and monoclonal reagents applied to Gram negative diplococci isolated from various anatomical sites are given in Table 3 . The values of a (sensitivity), b (specificity), and p (prevalence) used in calculating the predictive values given in Table 3 were derived as follows.

The sensitivity and specificity of the monoclonal reagent were $96.6 \%(198 / 205)$ and $98.2 \%(108 /$ $110)$ respectively; these figures represent all clinical isolates tested by the boiled suspension method. The sensitivity and specificity of the polyclonal reagent were 97.4\% (406/417) and 96.1\% (173/180), respectively. Because of the small number of isolates tested with the polyclonal reagent these are composite results of the present and previous study, ${ }^{5}$ which gave a sensitivity of $96.7 \%(318 / 329)$ and a specificity of $96.8 \%(120 / 124)$ with the same reagents.

The values of $p$ (the proportion of gonococcal Gram negative diplococci within the total population of Gram negative diplococci from various anatomical sites) as given in Table 3 were taken from the finding that over a 4 year period $N$ gonorrhoeae accounted for $20.3 \%$ of 1204 throat isolates of Gram negative diplococci ${ }^{6}$; and that of the Gram negative diplococci isolated over a 5 year period $N$ gonorrhoeae accounted for $99.6 \%$ of 1944 anogenital isolates from women, $99.7 \%$ of 2623 urethral isolates from heterosexual men, $95.3 \%$ of 384 anogenital isolates, and $92.0 \%$ of 225 rectal isolates from homosexual men."

\section{Discussion}

The advent of monoclonal antibodies raises the possibility of improved and more widely applied immunological methods of identifying microorganisms. In our "in use" evaluation, however, there was no significant difference in sensitivity and specificity of monoclonal and polyclonal antibody reagents for the routine identification of clinical isolates of $N$ gonorrhoeae.

Although factors such as sensitivity and specificity are important in evaluating new methods, the utility of a test result (the predictive value) depends on the prevalence of gonococcal Gram negative diplococci among the total population of Gram negative diplococci tested. As shown in Table 3, although the sensitivity and specificity of the test method remain the same, the predictive value varies greatly depending on the anatomical site of isolation. From these results we can make sound recommendations regarding the application of immunological methods for the laboratory identification of $\boldsymbol{N}$ gonorrhoeae.
Clearly, coagglutination testing with either polyclonal or monoclonal reagents may be recommended for the identification of gonococci from an anogenital site, although reliability is less in the case of isolates from homosexual men, when 1 to 3 per 1000 positive tests will be false positives; the corresponding rate with isolates from heterosexual patients is 1 to 2 false positives per 10000 positive tests. A positive coagglutination result on an isolate from the throat is less reliable than in the case of anogenital isolates; there will be 7 false positives per 100 positive tests with the monoclonal reagent and 14 false positives per 100 positive tests with the polyclonal reagent. Although this difference is not significant at the $5 \%$ level with 100 positive results, it becomes significant with 200 positive results.

It should be emphasised, however, that our criteria in assessing these reagents are extremely strict. Of 110 non-gonococcal neisseriae 108 gave unequivocally negative results, giving a specificity of $98.2 \%$. One isolate of $N$ lactamica gave a nonspecific reaction and one strain of $N$ meningitidis gave a positive reaction on first isolation but was negative when tested after two sub-cultures. In calculating the positive predictive value, these two results were included as "positive tests." It is of interest that these two results occurred in the first part of the survey, when we had least experience of the reagents; during the second part of the survey the specificity was $100 \%(54 / 54)$. In a preliminary evaluation of the same monoclonal reagents Philip et $a l^{12}$ found all 27 isolates of non-gonococcal neisseriae tested gave a negative reaction. Although a larger study is required, we consider that monoclonal coagglutination reagents are likely to achieve a level of specificity such that the predictive value of a positive test result is a reliable indicator of pharyngeal gonorrhoea in patients attending a clinic for sexually transmitted diseases.

Because of the predominance of meningococci in the throat a negative coagglutination result is a reliable indicator of non-gonococcal neisseriae; fewer than one negative result in $\mathbf{1 0 0}$ will be a false negative. Because of the extremely rare occurrence of meningococci in anogenital specimens from heterosexual patients, however, a negative coagglutination result is not a reliable indicator of nongonococcal neisseriae; about 9 of 10 negative results will be false negatives and gonococcal infection could pass undiagnosed. Although meningococci are isolated more frequently from anogenital sites in homosexual men, a negative coagglutination result with these isolates remains unreliable since 2 to 4 of 10 negative results will be false.

Because of its slightly greater sensitivity the negative predictive value of the polyclonal reagent is 
marginally better than that of the monoclonal reagent. A sensitivity of $99.99 \%$ would be required, however, to give a negative predictive value of $97.5 \%$ for an anogenital isolate from a heterosexual patient; a sensitivity of $99.90 \%$ would give a negative predictive value of only $79.7 \%$. It will obviously be difficult to achieve a sensitivity greater than $99.90 \%$ given that the sensitivity of the monoclonal reagent was $96.6 \%(198 / 205)$ in our study and is comparable to the figure of $96 \%(48 / 50)$ found by Philip et al. ${ }^{12}$

Monoclonal antibodies have been criticised as diagnostic reagents on the grounds that their very high specificity results in decreased sensitivity. To overcome this problem individual antibodies are normally combined to produce a broadly reactive reagent. Although the authors are unaware of the detailed characterisation of the monoclonal antibodies used in the GONO Gen reagent, other studies $^{13}$ suggest that suitable combinations of monoclonal antibodies will result in broadly reactive reagents with high sensitivity. The 16 monoclonal antibodies described by Tam et al ${ }^{13}$ recognise sub-group antigens rather than strain specific antigens, which may be distinguished by serotyping. ${ }^{14}$ One antibody (4-GS) identified an epitope shared by all 14 gonococcal reference strains containing the protein IA molecule whereas another monoclonal antibody (2-H1) detected an epitope shared by all 20 reference strains containing the protein IB molecule; the other 14 monoclonal antibodies recognised subsets of reference strains within the protein IA or IB groups.

Gonococcal serogroups WI and WII/WIII as

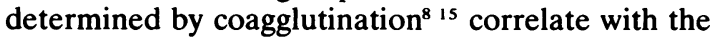
serotyping system of Buchanan and Hildebrandt ${ }^{14}$ based on antigenic differences in purified outer membrane protein I. Protein IA and IB are mutually exclusive forms of outer membrane protein I corresponding to serogroups WI and WII/WIII respectively. ${ }^{15}$ Serogroup WIII most probably represents a minor but prominent antigenic variant of protein IB rather than a specific moiety. ${ }^{8}$

Broadly reactive reagents containing combinations of monoclonal antibodies will require careful monitoring as the relative proportion of strains falling within a particular serogroup is likely to show considerable geographical and temporal variation. For example, of 195 gonococcal strains isolated from patients who acquired their infections in the Edinburgh area $44 \%$ belonged to serogroup WI and $56 \%$ to WII ${ }^{16}$ : in contrast, of six strains isolated from infections acquired outside the Edinburgh area three (West Africa one and Far East two) were serogroup WIII.

The use of a boiled suspension of organisms may help in the detection of a wide range of serotypes with the minimum number of monoclonal antibodies. Monoclonal antibodies reacted more strongly and gave additional positive reactions with boiled organisms when compared with unheated control suspensions, which suggests an increased accessibility of antibody to certain epitopes after boiling. ${ }^{13}$ Although autolytic conditions such as an alkaline $\mathrm{pH}(\mathrm{pH} 8.3)$ and the presence of divalent cation chelators have been reported to enhance the coagglutination reaction and give increased sensitivity, ${ }^{17}$ the use of the "antigen releasing agent" used in this study can not be recommended.

We conclude that a positive coagglutination test with either monoclonal or polyclonal reagents on Gram negative diplococci isolated from an anogenital site provides reliable identification of $N$ gonorrhoeae and no further tests are required. Monoclonal reagents are recommended when testing Gram negative diplococci isolated from the throat, and provided that $N$ gonorrhoeae has been isolated from an anogenital site a positive result is a reliable indicator of pharyngeal gonorrhoea and no further tests are required. Although negative coagglutination results with either polyclonal or monoclonal reagents need not be confirmed in the case of Gram negative diplococci isolated from the throat, it is mandatory to identify coagglutination negative isolates from anogenital sites by biochemical tests. Rapid carbohydrate utilisation tests ${ }^{18}$ would appear to be particularly useful for establishing the identity of such isolates. For laboratories not wishing to prepare the appropriate reagents, reagent impregnated neisseria identification discs are available commercially (Oxoid, Basingstoke).

Katherine G Reid gratefully acknowledges receipt of a Faculty of Medicine Research Scholarship.

\section{References}

' Department of Health and Social Security. Extract from the annual report of the Chief Medical Officer for the year $1980 . \mathrm{Br}$ J Vener Dis 1983;59:134-7.

${ }^{2}$ Danielsson D, Kronvall G. Slide agglutination method for the serological identification of Neisseria gonorrhoeae with antigonococcal antibodies absorbed to protein A-containing staphylococci. Appl Microbiol 1974;27:368-74.

${ }^{3}$ Rulfi T. Identification of Neisseria gonorrhoeae in the routine venereological laboratory. Comparative study of coagglutination, direct immunofluorescence, and sugar fermentation reaction. Br J Vener Dis 1980;56:144-7.

${ }^{4}$ Shanker S, Daley DA, Sorrell TC. A rapid slide coagglutination test-an alternative to the fluorescent antibody test for the identification of Neisseria gonorrhoeae. J Clin Pathol $1981 ; 34: 420-3$.

${ }^{5}$ Young H, McMillan A. Rapidity and reliability of gonococcal identification by coagglutination after culture on modified New York City medium. Br J Vener Dis 1982; 58:109-12. 
- Young H, Bain SSR. Neisserial colonisation of the pharynx. Br J Vener Dis 1983;59:228-31.

${ }^{7}$ McMillan A, Young H. Gonorrhoea in the homosexual man: frequency of infection by culture site. Sex Transm Dis 1978;5:146-50.

${ }^{8}$ Sandstrom EG, Knapp JS, Buchanan TM. Serology of Neisseria gonorrhoeae: $\mathrm{W}$ antigen serogrouping by coagglutination and Protein I serotyping by enzyme-linked immunosorbent assay both detect Protein I antigens. Infect Immun 1982;35:229-39.

' Young H. Identification and penicillinase testing of Neisseria gonorrhoeae from primary isolation cultures on modified New York City medium. J Clin Microbiol 1978;7:247-50.

${ }^{10}$ Veehio TJ. Predictive value of a single diagnostic test in unselected populations. N Engl J Med 1960;274:1171-3.

1 Young H. Characterisation of pathogenic neisseriae. Symposium on practical aspects of microbial characterisation in relation to microbiology. Pathological Society of Great Britain and Ireland. 1983.

12 Philip SK, Ison CA, Easmon CSF. Coagglutination identification of Neisseria gonorrhoeae (Letter). Br J Vener Dis 1984;60:66.

${ }^{13}$ Tam MR, Buchanan TM, Sandstrom EG, et al. Serological classification of Neisseria gonorrhoeae with monoclonal antibodies. Infect Immun 1982;36:1042-53.
${ }^{14}$ Buchanan TM, Hildebrandt JF. Antigen-specific serotyping of Neisseria gonorrhoeae: characterization based upon principal outer membrane protein. Infect Immun 1981;32:985-94.

is Sandstrom EG, Chen KCS, Buchanan TM. Serology of Neisseria gonorrhoeae: Coagglutination serogroups WI and WIIWIII correspond to different outer membrane Protein I molecules. Infect Immun 1982;38:462-70.

${ }^{16}$ Reid KG, Young H. Serogrouping of Neisseria gonorrhoeae: correlation of coagglutination serogroup WII with homosexually acquired infection. Br J Vener Dis 1984 (in press).

17 Izakson I, Morse SA. Enhancement of coagglutination reactions of the Phadebact Gonococcus Test by ethylenediaminetetracetate and ethylene glycol-bis (B-aminoethyl ether)-N,N-Tetraacetate. J Clin Microbiol 1981;14:261-65.

${ }^{18}$ Tapsall JW, Cheng J. Rapid identification of pathogenic species of Neisseria by carbohydrate degradation tests. Importance of glucose in media for preparation of inocula. $\mathrm{Br} J$ Vener Dis 1981;57:249-52.

Requests for reprints to: Dr H Young, STD Diagnostic Laboratory, Department of Bacteriology, University of Edinburgh Medical School, Teviot Place, Edinburgh EH8 9AG, Scotland. 